\title{
Experiencias de las familias creole en el rescate y revitalización de su identidad colectiva en contex- tos multiculturales
}

\author{
Creole families experiences in the rescue and revitalization of their collective identity in multicultural \\ contexts
}

\author{
Sandra Carolina Rojas Hooker' \\ Loyda María Stamp Lackwood²
}

\section{Resumen}

Este artículo se basó en el estudio titulado: "Experiencias de las familias creole en el rescate y revitalización de su identidad colectiva en contextos multiculturales" se han analizado las dinámicas de convivencia y socialización predominante en la familia Creole que contribuyen al fortalecimiento de su identidad. La metodología que se utilizo es cualitativa, de corte transversal, descriptivo fenomenológico encaminado al reconocimiento de situaciones y comportamientos mediante la historia oral para identificar valores, creencias, normas y prácticas del pueblo afrodescendiente mediante la interacción con los participantes.

Las participantes identificaron como el principal factor de debilitamiento de la identidad creole la no utilización del creole como lengua materna en la familia, escuela y la iglesia, principalmente en niños, adolescentes y jóvenes debido a una alta migración de la población creole, la reconfiguración de la población del barrio y sobre todo la no implementación del modelo de familia basado en valores.

Palabras clave: Afrodescendientes; cultura; familia; rescate; revitalización.

\section{Abstract}

This article was based on the study "Types of property ownership in the Caribbean Coast of Nicaragua" with an emphasis on the complexity and types of conflict in property ownership. For this, the different types of property are analyzed based on the principles and categories contained in the Magna Carta, the general and special laws related to this subject. The methodology was qualitative aimed at recognizing the levels of complexity and types of conflict that occur according to the forms of ownership recognized in national legislation. For this, focus groups and interviews were carried out with the participation of representatives of the Municipal Governments, representatives of indigenous and Afro-descendant governments, and other social sectors, as well as a documentary review in order to have a comprehensive look at this issue.

The main complexities are the growing non-indigenous population in indigenous territories, the politicization and partisanship in the election of community authorities, the lag and the lack of completion of the sanitation phase that prevents the issuance of property titles in favor of the indigenous communities and consequently makes investment negotiations difficult.

Keywords: Property Ownership; forms of ownership; collective rights; complexity levels; types of conflict.

\footnotetext{
1 Máster en Estudios Indígenas. Directora Instituto de Estudios y Promoción de la Autonomía de la Universidad de las Regiones Autónomas de la Costa Caribe Nicaragüense. Correo: sandra.rojas@uraccan.edu, ni; No. ORCID https://orcid.org/0000-0002-7467-2366

2 Máster en Salud Intercultural. Coordinadora de Interculturalidad de la Universidad de las Regiones Autónomas de la Costa Caribe Nicaragüense. Correo: loyda. stamp@uraccan.edu.ni; No. ORCID https://orcid.org/0000-0003-0489-979X
} 


\section{Introducción}

Este trabajo tiene el propósito de compartir algunas dinámicas de convivencia y socialización predominante en la familia creole que contribuyen al fortalecimiento de la identidad. Lo anterior nos permitirá conocer y valorar aún más la riqueza cultural al compartir algunos principios y valores que dinamizan esas relaciones afectivas y de socialización, así como las formas de transmisión de conocimientos, saberes y prácticas propias. Estas tradiciones se encuentran en la memoria colectiva y consideramos necesario plasmarlo en el presente trabajo para su práctica, conocimiento y perpetuidad.

La familia está presente en la vida social. Es la más antigua de las instituciones humanas y constituye el elemento clave para la comprensión y funcionamiento de la sociedad. A través de ella, "la comunidad no sólo se provee de sus miembros, sino que se encarga de prepararlos para que cumplan satisfactoriamente el papel social que les corresponde. Es el canal primario para la transmisión de los valores y tradiciones de una generación a otra" (Gustavikno 1987:13)

Las entrevistas y grupo focal nos permitió tener un registro de la comunicación de las personas, su lenguaje y formas propias de interpretar sus formas de convivencia mediante la cual pudimos reconocer las significaciones que le dan a hechos y plasmar en un escrito las vivencias, experiencias y sentimientos y compartir con los pueblos indígenas, afrodescendientes, mestizos y comunidades algunos principios, valores y mecanismos que son necesarios implementar para el rescate y revitalización de la identidad colectiva del pueblo afrodescendientes.

\section{Revisión de la literatura}

Para los afrodescendientes la familia es un factor clave para la reproducción, la conservación, la solidaridad y la resistencia como pueblo. Es el espacio primario de socialización, donde más allá del padre, la madre y los hijos se consideran también parte de ella: tíos, primos, abuelos, y otros afines, todos con deberes y derechos definidos. Además, la comunidad permanece integrada bajo un concepto de familia más amplio; todos son una familia y estas relaciones de parentesco son fundamentales para la conservación de su identidad (Cantillo 2013: 197, 198).

La identidad étnica es una construcción social de carácter configurante, que difiere de un lugar a otro. Es decir, no debe ser comprendida como algo esencial. Es preciso entenderla como un proceso afectado por la historia y las circunstancias contemporáneas, tanto por la dinámica local como por la global.

Los afrodescendientes somos el resultado de una diáspora que tuvo inicio hace cinco siglos, pero pervive su legado a través de las tradiciones culturales y dinámicas políticas en las que están incursionando. Lo importante es entender que la identidad étnica se convierte en un fenómeno de movilización y autoafirmación positiva, tanto para el individuo como para la colectividad.

Por la compleja diversidad de orígenes en las culturas africanas, hoy, de manera consciente y autónoma, reconstruimos y asumimos nuestras raíces y herencia de las culturas africanas ancestrales, y construimos el concepto de nuestra identidad cultural o étnica colectiva: el afrodescendiente, afro- nicaragüense, negros, creole y negro-creoles con una historia en común: Somos descendientes de los africanos que fueron secuestrados en África y traídos como esclavos a las Américas. Siendo este el comienzo de una larga línea de represión, invisibilización, de esclavización, pero también, una historia de resistencia, de construcción de nación y de elaboración de propuestas para una mejor sociedad.

Hoy día, los afrodescendientes hemos reivindicado nuestra pertenencia nacional e identidad a partir del reconocimiento e inclusión como pueblos en la Constitución Política y demás leyes. Es el resultado de un proceso histórico en el que interactúan diversos actores que convergen en una constante dinámica social que tiene como fuerzas motoras la familia, la comunidad, la iglesia, entre otros. También intervienen 
otros factores, como señala Alejos (2004:1) y es el temor a la pérdida de identidad propia, o a ser objeto de la imposición de identidades ajenas anima las más heterogéneas reacciones individuales y colectivas.

La identidad es una necesidad para la existencia y desarrollo de los pueblos con el fin de establecer su propio ser en el proceso de interrelación con otros pueblos, sin perder su propia identidad; permite el reencuentro entre los pueblos que vitaliza y dimensiona con mayor fuerza la cultura a través de la práctica diaria.

De acuerdo a García (1992:247-248) en la conformación de las identidades, la historia juega un papel importante y esto se señala en cuando la historia es también un lugar de identificación, es en ella que se organiza la continuidad estructural del sujeto y de la formación social, [...] la historia personal y social de los individuos y de las sociedades, sus sistemas de valores, su capacidad de suscitar la adhesión, etc. son componentes inalienables de la herencia social.

La identidad supone un ejercicio de autoreflexión, a través del cual el individuo pondera sus capacidades y potencialidades, tiene conciencia de lo que es como persona; sin embargo, como el individuo no está solo, sino que convive con otros, el autoconocimiento implica reconocerse como miembro de un grupo que comparten valores, actitudes, estilos de vida, costumbres y ceremonias; lo cual, a su vez, le permite diferenciarse de los miembros de otros grupos.

Para Henry Tajfel la pertenencia al grupo es el ingrediente esencial de la identidad social, porque al mismo tiempo que se siente parte de un grupo, el individuo se diferencia de los miembros de otros grupos a los que no pertenece; por ello se dice que la fuente de identificación del individuo es el propio grupo, pero los otros juegan también un papel importante, ya que cuando experimenta que es diferente a los otros se reafirma la pertenencia al grupo (Ibid., 2010: 231).

\section{Materiales y métodos}

La metodología que se utilizó es cualitativo, de corte transversal, descriptivo fenomenológico encaminado al reconocimiento de situaciones y comportamientos mediante la historia oral para identificar valores, creencias, normas y prácticas del pueblo afrodescendiente mediante la interacción con los participantes. Se realizó grupos focales, entrevistas donde participaron diferentes personalidades, sectores sociales y religiosos, jóvenes, adultos y ancianos de ambos sexos.

Los resultados fueron leídos, analizados y organizados en base a las etapas e hitos del duelo Afrodescendiente que inician con las señales o anticipos, fallecimiento y velorio en casa, entierro, nueve días y culmina en algunos casos con la ritualidad de año.

\section{Resultados y discusión}

\section{Origen y procedencia de los creoles en Puerto Cabezas}

El origen de la población creole parece ser muy diverso. Romero (2007:73) menciona que la población creole que se asentó en la Costa Caribe Nicaragüense es de descendencia africana. Llegaron a la región por diferentes circunstancias: debido a naufragios de barcos esclavos en el mar Caribe, de sublevaciones de esclavos que escapaban y buscaban refugio en la región, y también como esclavos de los colonos británicos desde la primera mitad del siglo VII; otros se establecieron en la región en su condición de hombres libres.

Bush, Martin y Rigby (2000:17) señalan que la llegada de los creoles a Puerto Cabezas está estrechamente vinculada al establecimiento de las primeras transnacionales y la demanda de mano de obra calificada para las actividades de extracción minera, forestal y bananera desde los años 1926.

La actual población creole de Bilwi tiene sus raíces en Bluefields, Laguna de Perlas, Belice, Honduras, Jamaica y Haití entre otros lugares. La población creole es el resultado de una diáspora que tuvo inicio 
hace cinco siglos, pero pervive su legado a través de las tradiciones culturales y dinámicas políticas en las que están incursionando.

\section{Identidad colectiva y entorno sociocultural}

La identidad es un sistema cultural de referencia, a partir del cual una comunidad define su identidad grupal. La identidad social es el reconocimiento por parte del individuo de su pertinencia a un grupo social, este reconocimiento trae implícito su significado emocional y valorativo de su pertenencia al grupo (Aguirre, 2006).

$\mathrm{Al}$ consultar a los participantes sobre su auto adscripción étnica la gran mayoría se identifica como mujer u hombre creole, black o black-creole mediante la construcción de la identidad colectiva: El afrodescendiente.

Una de las participantes del grupo focal señala que el término afrodescendiente es un término político que se utiliza en el sistema de Naciones Unidas al momento de elaborar las diferentes declaraciones, convenios y tratados para englobar a todas aquellas personas que tienen un ancestro común de África, pero que en nuestro entorno social debemos de identificarnos por el nombre al cual hemos designado a la colectividad: creole.

En palabras de una de las entrevistadas:

El término afrodescendiente es reciente, y todavía siento que hay una discusión alrededor de que si es aceptado o no por la comunidad negra que vive aquí en Puerto Cabezas. Pero reconocemos que está relacionado a la esclavitud y a un ancestro común que vino de África.

Lo antes expresado por las personas entrevistadas y grupo focal se corrobora con lo dicho por Barth y Stavenhagen al señalar que entre las características esenciales que deben de reunir las colectividades para ser considerados una comunidad destacan:

1. Valores culturales fundamentales compartidos y realizados con unidad manifiesta en formas culturales.

2. Integra un grupo de comunicación e interacción.

3. Cuenta con miembros que se identifican a sí mismo y son identificados por otros y que constituyen una categoría distinguible de otras categorías del mismo orden.

La etnicidad es un producto del proceso de identificación que puede definirse como la organización social de la diferencia cultural. Por lo tanto, lo que realmente importa para explicar la etnicidad no es tanto el contenido cultural de la identidad, sino los mecanismos de interacción que, utilizando cierto repertorio cultural de manera estratégica y selectiva, mantienen o cuestionan las fronteras colectivas ${ }^{3}$. Hacen referencia sus prácticas como un todo que los identifica al definirlo como el conjunto de conocimientos, saberes, creencias, costumbres, valores, las prácticas cotidianas que va desde la manera de comportarse hasta la manera en como educan a las nuevas generaciones.

La mayoría de las mujeres refieren que la cultura que impera en su familia es la suya porque son enseñanzas inculcadas desde muy temprana edad y que transmiten a sus hijos desde pequeños a través del idioma, vestimenta, alimentos, expresiones culturales y los valores. El hecho de que los padres sean afrodescendientes refuerza la auto adscripción al señalar con firmeza: "porque mis padres lo son y bajo esa cultura fui formada".

\footnotetext{
3 Véase: Barth, Frederik (1976). Los grupos étnicos y sus fronteras. México y Stavenhagen, Rodolfo (1996). Ethnic Conflicts and the Nation State. Mc Millan Press LTD. London
} 
Sobre ese particular, las entrevistadas señalan:

Ser una familia Creole está relacionado a tu comida, tus costumbres y tus actividades culturales y que son diferentes al resto. Son prácticas, valores y hábitos relacionadas intrínsecamente con la educación como un elemento que destaca en la cultura Creole.

El concepto de familia en los Creoles considero que no se parece al que tienen otros pueblos o comunidad étnica porque el elemento más fuerte que los identifica es el lenguaje como punto de partida. Luego, las prácticas culturales que son distintas, el modo de asentamiento, la construcción de la vivienda y los miembros de la familia compuesta generalmente por mama, papa, abuela, hijos.

Principios y valores que dinamizan las relaciones afectivas y de socialización de la familia creole

La familia como institución social es producto de la sociedad que la crea, perpetúa e igualmente, la regula. Se reproduce en el interrelacionar humano de la vida cotidiana que una sociedad establece en un contexto de tiempo y espacio cultural, económico y político determinado.

Para los afrodescendientes la familia es un factor clave para la reproducción, la conservación, la solidaridad y la resistencia como grupo. Es el espacio primario de socialización, donde más allá del padre, la madre y los hijos se consideran también parte de ella: tíos, primos, abuelos, y otros afines, todos con deberes y derechos definidos. Además, la comunidad permanece integrada bajo un concepto de familia más amplio; todos son una familia y estas relaciones de parentesco son fundamentales para la conservación de su identidad (Cantillo 2011: 197, 198).

Al indagar sobre los principios y valores coinciden en que se han inculcado en el seno familiar el respeto principalmente hacia los mayores, la potestad del vecino de corregir algún comportamiento de los menores que estaba fuera de las normas morales y sociales. Concuerdan que el respeto es un elemento integral en la vida constituyéndose en un elemento clave para la comprensión y funcionamiento de la sociedad. Reafirman sin distinción que los principios y valores se inculcan en la casa, la familia y por ello cuando algún miembro las transgrede ocasiona un malestar no solo a su familia, sino también a toda la comunidad creole.

Estos valores son el respeto, sinceridad - veracidad, solidaridad, cortesía, consideración, tolerancia y responsabilidad. Señalan que dentro de los valores que le fueron transmitidos de generación en generación destacan:

1. El amor al prójimo

2. El respeto a los adultos, respeto a uno y a las personas que lo rodean.

3. Ayuda a los demás miembros de la familia y otras personas en momentos de necesidad, el compartir con los vecinos los productos cosechados, horneados o un platillo especial los fines de semana.

4. Unidad y la solidaridad presente en las celebraciones de fechas especiales, problemas de salud y muerte.

5. Cultura de no violencia, respeto a la propiedad ajena" aprender a vivir con lo que uno tiene, no codiciar los bienes del vecino, amigo, conocido".

6. La responsabilidad familiar y social en cuanto al buen comportamiento del menor y el llamado de atención por un adulto, sin que esto cause malestar a la familia, siendo la misma un mecanismo de control social y educación colectiva. 
7. Priorización de las necesidades de los miembros de la familia empezando por el adulto mayor y menores de edad.

8. Los buenos modales y la cortesía "buenos días, buenas tardes, buenas noches, con permiso, por mencionar algunos.

Sobre este particular el líder religioso rememora:

Nosotros no aprendimos a ser violentos, no aprendimos a robar. Aprendimos a obtener las cosas de manera correcta. Debemos de rescatar esos valores ya que es triste ver hoy día, personas del pueblo creole involucrados en drogas, en crímenes, encarcelados. Hace treinta años no se miraba esto, hoy, nos ha invadido ¿Que nos está pasando? Estamos perdiendo los principios y valores como Creole, principios y valores que nuestros padres nos enseñaron.

Las entrevistadas afirman que la solidaridad era un principio básico del pueblo creole, solo debíamos tener conocimiento que alguien necesita ayuda, que algo le pasa, teníamos ese apoyo. Ahora se observa más en el ámbito de la familia nuclear y familia ampliada. Sumado a esto, el apoyo y la solidaridad se dan de manera individual y particularizada, no como colectividad.

Existe un reclamo explícito y un llamado al pueblo creole sobre la no la transmisión de valores de manera sistemática a la siguiente generación, reconociendo también que es obligación única y exclusiva de los miembros de la familia. A la vez, reconocen que las generaciones de jóvenes actuales no están siguiendo los patrones culturales de sus padres o abuelos. Existe una preocupación generalizada al considerar que los valores considerados propios del pueblo creole van en desuso sumado a una creciente rebeldía en los jóvenes.

Algunos participantes al referirse al tema de cómo se manifiesta la unidad en los espacios de participación colectiva señalan:

El común denominador de toda esta problemática no somos unidos, constantes ni pacientes. Si te niegan apoyo en un primer momento dejamos de insistir, ya no regresamos a esas instancias porque somos orgullosos, somos pobres pero orgullosos hasta el punto de dejar de comunicarnos con otras personas de nuestra comunidad en caso de tener una desavenencia.

La unidad como condición esencial para la armonía y el entendimiento en un grupo, reúne al pueblo creole en torno a objetivos y creencias comunes y se construye a partir de una visión compartida para el bien colectivo. No obstante, en el contexto actual se reconoce que este valor ha ido debilitándose dada la reconfiguración de los barrios, el contexto multiétnico y sobre todo la emergencia de organizaciones sociales afrodescendientes diversos con agendas que no corresponden a las necesidades y prioridades del pueblo afrodescendiente.

Los participantes externaron sus preocupaciones sobre la necesidad de contar con organizaciones sociales que contribuyan al fortalecimiento de la identidad creole, con representantes y estructuras gubernamentales que elaboren y pongan en práctica políticas, planes, programas y proyectos con miras a cumplir los objetivos del Decenio Internacional de los Afrodescendientes, entre estos, la plataforma política que nos ayudará a combatir la exclusión y marginación que nos afecta, permitiéndonos ser sujetos de nuestra propia transformación.

\section{Espacios de socialización que dinamizan la identidad creole}

En la Costa Caribe Norte, la mayoría de las familias pertenecen y se identifican con la Iglesia Morava por haber sido ésta una institución que influyó poderosamente en la comunidad negra costeña (siglo XVIII), ofreciendo oportunidades educacionales y el único programa de salud y bienestar social en ese entonces. Se suman a este proceso las iglesias Anglicanas, Bautista y Adventista con la finalidad de conjugar los valores 
cristianos con la transmisión de la cultura como una dimensión espiritual de su identidad afro reforzando la importancia de valores como el respeto, responsabilidad, honestidad entre otros, que son de mucha importancia para la convivencia, la paz y armonía social.

Sobre este particular, los entrevistados realizaron una reflexión autocritica sobre el papel de estas instituciones sociales en la conducción individual y colectiva de los creoles al señalar que la Iglesia no debe limitarse a trabajar la parte espiritual de los feligreses, sino también debe de enfocarse en la enseñanza de los valores humanos y, sobre todo, fortalecer los rasgos identitarios, entre estos el idioma materno. Así, existen posiciones encontradas por parte de los entrevistados al señalar que la responsabilidad mayor en la preservación de la cultura recae básicamente en la familia y que la función principal de la iglesia es predicar la palabra de Dios de manera inclusiva mediante la utilización de los idiomas de las personas presentes.

Sobre este particular, un líder religioso opina:

La iglesia no puede fortalecer tu identidad étnica. lo único que puede hacer la iglesia es fortalecer la espiritualidad del ser humano, no importa tu raza o sexo, que tan grande es tu fe. en mi iglesia, todos son bienvenidos, no importa qué idioma hablen, ya que desarrollamos el culto en creole, español y miskito. La iglesia no debe de hacer diferencia entre los diferentes grupos étnicos.

Identificamos elementos esenciales planteados por Talcott Parson al reconocer que la familia es, junto a la escuela y la religión, el vehículo esencial de la socialización en general, y lo es también de la socialización política, en particular. Es la primera institución socializante individual y colectiva de los seres sociales para formar el hombre y mujer que la sociedad requiere para su funcionamiento y mantenimiento ${ }^{4}$.

Se observa que hay posiciones diversas sobre la utilización del idioma creole para las actividades propias de la iglesia. Consideran que el rol de la iglesia es acercar a las personas al Ser Supremo a través del fortalecimiento de su espiritualidad. Se brinda apertura a que los diferentes pueblos y comunidades participen en dichos servicios religiosos es necesario la utilización del idioma español y creole a través de un servicio mixto ya que la mayoría de los niños y jóvenes que visitan las iglesias no dominan el creole y que se sientan parte de la congregación se ha optado por la realización de actividades en los idiomas en que puedan expresar su espiritualidad a través de las alabanzas y lecturas bíblicas.

Existe una necesidad inminente de hacer mayor énfasis y utilizar mecanismos que atraigan a los jóvenes para empezar a hablar el idioma creole y garantizar de esta manera la preservación de la cultura vinculado intrínsecamente al aspecto religioso e identitario como partes fundamentales de su identidad.

Sobre el rol de la escuela en la preservación y transmisión de la cultura consideran a pesar de que la mayoría de las iglesias todavía bajo su conducción Centros de Educación Primaria y Secundaria debería de abonar al esfuerzo de la formación y puesta en práctica de la Educación Intercultural Bilingüe a través de la implementación del programa educativo en ingles - español.

La enseñanza del inglés es visto como una oportunidad de mercado para los jóvenes costeños. No obstante, no hay un posicionamiento claro si esta educación debe de realizarse en creole-español o inglés-español señalando algunos de los entrevistados que el aprendizaje del inglés standard no abona al fortalecimiento y preservación del creole como idioma materno.

Para la familia creole el barrio constituye un espacio de socialización importante, principalmente en la etapa de la niñez y adolescencia ya que contribuye a la consolidación de la identidad colectiva. Una de las entrevistadas rememora y comparte con nosotros su experiencia como madre al decir:

Tres de mis cuatro hijos hablan el Creole, ya que lo vecinos eran mayoritariamente Creoles y los niños a través de los juegos y cuentos infantiles, hicieran uso de su lengua materna. Sin embargo,

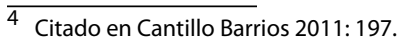


en la Década de los Ochenta productos de la migración masiva de familias Creoles, su hija menor no tuvo esa oportunidad, hablando hoy día solamente el español y entendiendo algunas palabras en el inglés.

Lo anterior se refuerza con lo dicho por John Holms (1968) al señalar que la perdida de la habilidad para hablar creole es a menudo suficiente para no ser considerado creole por otros creoles ${ }^{5}$. Se observa que el elemento más fuerte es el lenguaje como punto de partida y que esta se fortalece desde la casa.

Valorando la influencia de los medios de comunicación oral y escrita en un contexto globalizado, reconocen el rol que juegan los programas de TV en la vida de los niños, adolescentes y jóvenes, ya que pasan gran cantidad de tiempo viendo programas diversos en el idioma español.

Debido a lo anterior, se hace necesario tener programas educativos y de entretenimiento en el idioma ingles, tomando en cuenta la diversidad étnica y cultural que existe en la región. A la vez instan a los medios nacionales, principalmente el Canal 6 de transmitir al menos ciertos programas en nuestro idioma materno. Ante este panorama, consideran los participantes que debemos de reforzar nuestro rol como transmisoras de la cultura.

\section{El rol de la familia en el rescate y revitalización de la identidad creole}

La familia matriarcal posee determinadas características en cuanto a su organización, su origen y sus formas, que la distinguen de otros tipos de organización familiar.

La influencia femenina era muy grande en la sociedad, ya que la mujer ejerce el poder político, religioso y económico. La mujer creole es firme en su carácter, cuida a su familia, se encarga de la salud, educación y espiritualidad de sus hijos. Es la jefa, la que lidera las diferentes personas, tienen iniciativa, son decididas y son valientes. En palabras de una de las entrevistadas:

"La mujer negra tiene una fuerza de liderazgo y dominio nato, lo va inculcando a sus hijas en los espacios que comparten como los quehaceres de la casa y educación. Aunque está casado con alguien de otra etnia, predomina en la mayoría de los casos el sistema matriarcal".

En la familia creole se reconoce el rol preponderante que desempeñan las mujeres, principalmente las abuelas en la formación y fortalecimiento de la identidad colectiva. Ya que cohesiona la cotidianidad de la parentela. La mujer es orientadora, confidente, guiadora, motivadora, autoafirmadora y cuida que su descendencia conserve y perpetúe las tradiciones prácticas y simbólicas valoradas desde lo ancestral. Las mujeres, especialmente las abuelas son garantes de la memoria colectiva viviente de la función familiar en el contexto de su cosmovisión; consideradas depositarias y transmisoras de la identidad colectiva.

Las participantes con mucho orgullo señalan:

"Debemos de platicar sobre la importancia de mantener la identidad. Comencé a hablarle a mi nieta en creole, regresar a lo que practicaba mi mama, ya que afuera está obligada a utilizar otro idioma".

"We as Creole should bring up our children the way they taught us"6.

"Mi abuela me ha enseñado a ser fuerte, a ser resiliente, a superar los desafíos que se presentan en la vida y que con la ayuda de Dios podemos salir adelante".

\footnotetext{
5 Citado en los afrodescendientes (Creoles) y la revolución. Wani, julio - septiembre 1986.

6 Traducción propia: "Nosotros como Creoles debemos de criar a nuestros hijos de la forma que nos enseñaron nuestros Ancestros.
} 
"El idioma es una parte fundamental para que el pueblo creole para que nos sintamos orgullosos de nuestra herencia, para no perder nuestra memoria histórica y colectiva".

A través de estas voces valoramos que en la cultura creole aun predomina el sistema matriarcal basado en un conjunto de relaciones centrada en la figura de la madre, donde la mujer que es bisabuela y madre es el centro de su red consanguínea, la persona a través de quien pasa la descendencia y herencia, y quien ejerce el poder sobre su casa y familia. Es el principal foco difusor, a partir del cual se multiplican las relaciones entre todos los miembros de su red de parentesco, que generalmente va más allá de los límites físicos de su unidad doméstica en cuanto local especifico de residencia.

\section{Conclusiones}

Los entrevistados y participantes del grupo focal se identifican como mujer u hombre creole, black y una persona como black-creole mediante la construcción de la identidad colectiva: El Afrodescendiente con una historia en común: somos descendientes de los africanos que fueron secuestrados en África y traídos como esclavizados a las América. Identidad que asumen con mucho orgullo como una enseñanza de sus abuelas, madres, tías, hermanas mayores.

1. Los Creoles tienen un concepto muy integral de su identidad que abarca la religión, cultura compartida, un nombre en común que los identifica como colectividad, el idioma y un origen común y asumen con mucho orgullo la identidad creole como una enseñanza de sus abuelas, madres, tías y hermanas mayores.

2. Reconocen que la familia tiene un valor relevante en la construcción y fortalecimiento de la identidad la cual se manifiesta fuertemente en la educación, valores y principios, religiosidad y gastronomía. Hombres y mujeres participantes del estudio valoran el papel preponderante que tiene la mujer creole en la construcción de la identidad y transmisión de la cultura la cual asume de forma natural y no como una carga familiar y que es una herencia de sus ancestros, principalmente las abuelas.

3. Se percibe una profunda añoranza de educar a los hijos e hijas con los valores y principios tales como el respeto, honradez, cortesía, tolerancia y responsabilidad, unidad, solidaridad y cultura de no violencia y se plantean como desafíos retomar los modelos de crianza y modelos educativos basados en valores tanto en el ámbito privado como público, ya que la educación es el vehículo fundamental para mantener viva nuestra identidad colectiva, herencia de nuestros ancestros.

4. Los participantes enfatizaron sobren la importancia de la unidad y solidaridad como principios básicos de la cultura creole. Estos principios un persisten en el ámbito familiar. No obstante, en el ámbito colectivo aflora únicamente ante una situación de emergencia, enfermedad o duelo.

5. Las participantes identificaron como el principal factor de debilitamiento de la identidad creole la no utilización del creole como lengua materna en la familia, escuela y la iglesia, principalmente en niños, adolescentes y jóvenes debido a alta migración de la población creole, la reconfiguración de la población del barrio y sobre todo la no implementación del modelo de familia basado en valores. Sumado a eso, la falta de un programa educativo bilingüe intercultural que fortalezca el creole como lengua materna y el rol pasivo de las instituciones religiosas en promover el uso del creole en sus actividades religiosas.

6. El pueblo creole está consciente de la necesidad de la unidad y solidaridad representada por una estructura organizada con el objetivo de velar por los intereses de las familias. Ello permitirá unir fuerzas para realizar actividades de rescate de su cultura e identidad colectiva en contextos multiculturales. Para ellos es crucial la facilitación de espacios de participación y reuniones frecuentes de los miembros de la etnia, para crear armonía, comunicación fluida y conciencia, entre los miembros de la etnia, para fortalecer su identidad. 
1.

\section{Agradecimiento}

Esta publicación obtuvo el financiamiento de: El Fondo de Asistencia Internacional de los Estudiantes y Académicos Noruegos (SAIH).

\section{Lista de referencias}

Aguirre, Ángel (2006). Cultura e identidad cultural. España, Ediciones Bárcenas SL

Alejos García, José. (2004). Identidad étnica y conflicto agrario en Chiapas, Amérique Latine Histoire et Mémoire, Número 10.

Bush, Deborah; Martin, Elisa; Rigby, Betty (2000). Los Creoles de Bilwi en el proceso de autonomía costeña. URACCAN, No publicado.

Cantillo Barrios, Ligia (2013). Tradición cultural y familia afrodescendiente en Barranquilla. Revista Amauta, Universidad del Atlántico, Barranquilla, Colombia. No22, Julio - Diciembre 2013.

García, Jesús. (1992). Historias de nuestra historia. La construcción social de las identidades mayas en Guatemala. Ediciones Estipaz, Guatemala.

Gustavikno, E. (1987). Derecho de Familia Patrimonial. Bien de Familia. Tomo I. Segunda Edición. Argentina. 